\title{
EL ESPACIO ABIERTO: UNA TECNICA DIDACTICA FACILITADORA DEL DESARROLLO DE COMPETENCIAS GENERALES EN LA FORMACION PROFESIONAL SUPERIOR ${ }^{1}$
}

\author{
Alejandro Villalobos Claveria* \\ Yenia Melo Hermosilla**
}

Aprovado em: 29 de junho de 2009

* Dr. en Educación. Profesor del Depto. Ciencias de la Educación. Facultad de Educación. Universidad de Concepción, Chile. E-mail: avillalo@udec.cl

** Dra. en Educación. Profesora del Depto de Curriculum e Instrucción. Facultad de Educación. Universidad de Concepción, Chile. E-mail: ymelo@udec.cl

Resumen: El propósito principal del presente artículo es demostrar que el Espacio Abierto (Open Space) como técnica investigativa puede ser un adecuado procedimiento didáctico para el desarrollo de competencias genéricas (transversales) en la formación profesional superior. Para alcanzar dicho propósito, en primer lugar, se describe el Espacio Abierto como la técnica investigativa y la información requerida para su aplicación. En segundo lugar, se presenta una propuesta de aplicación ya validada con un conjunto de académicos de dos universidades regionales, para su eventual utilización en las aulas universitarias. El presente trabajo se centra en los resultados preliminares del proyecto Fondecyt $\mathrm{N}^{\circ}$ 1070466: "La construcción del sujeto docente universitario en universidades regionales: Factores claves en su desempeño académico y profesional”, financiado por el gobierno de Chile, en la línea Conicyt/Fondecyt. Estudio que pretende caracterizar factores claves que definen la profesión docente universitaria en instituciones de enseñanza superior regionales para el diseño de una propuesta de perfeccionamiento de académicos e investigadores en docencia superior.

Palabras clave: Educación superior. Didáctica. Formación del sujeto. Profesor universitario.

\section{THE OPEN SPACE: A FACILITATING DIDACTIC TECHNIQUE IN THE DEVELOPMENT OF GENERERAL COMPETENCES IN HIGHER EDUCATION}

\begin{abstract}
The main goal of this article is to demonstrate that the Open Space as a research technique can be a suitable didactic procedure for the development of general competences (cross-sectional) in higher education. In order to reach this goal, we begin by describing the Open Space as a research technique as well as the information required for its application. Secondly, an application proposal is presented, which has been previously validated by a group of professors from two regional universities. This proposal is intended to be used in university classrooms when possible. This study focuses on the preliminary results of Fondecyt Project $\mathrm{N}^{\circ}$ 1070466: "The education of the university professor in regional universities: Key factors in their academic and professional performance", financed by the government of Chile, along the Conicyt/Fondecyt lines. This study tries to characterize key factors that define the university teaching profession in regional higher education institutions and later on to design an improvement proposal for professors and researchers in higher education.
\end{abstract}

Key words: Higher education. Didactics. Education of the individual. University Professor.

1 Trabajo con resultados preliminares de Proyecto Fondecyt nº 1070466: "La construcción del sujeto docente universitario en universidades regionales: Factores claves en su desempeño académico y profesional”, financiado por el gobierno de Chile. 


\section{Introduccion}

De manera general, se puede afirmar que en todo proceso de Enseñanza - Aprendizaje, tanto los recursos y los medios de enseñanza, como la didáctica y el clima socio emocional del aula, constituyen factores claves en la interacción profesor - alumno.

Desde el comienzo de la pedagogía, la acción pedagógica se ha preocupado de encontrar unos recursos, medios y estrategias para mejorar la enseñanza. Habitualmente, la relación alumno - contenido - profesor se ha organizado en torno a algún procedimiento didáctico que facilite la labor formativa educadora en el aula. En este sentido, la situación del aula se transforma en un escenario de aprendizaje, donde la pizarra, la exposición del profesor, la observación, la guía didáctica, el texto de apoyo, el trabajo grupal, la lectura silenciosa, la investigación, entre otros recursos y medios que pueden formar parte del diseño y aplicación de alguna estrategia didáctica que potencie la labor educadora del profesor.

Este desafío permanente del profesor, independiente de su nivel de enseñanza, disciplina o calidad de los alumnos, se convierte en una tarea mayor cuando se trata de cursos numerosos y sobre todo, cuando se refiere a la formación profesional superior. Motivo por el cual, el presente trabajo intenta ofrecer una técnica de investigación cualitativa que, se transforma en una nueva herramienta didáctica para el docente universitario, quien, en ocasiones, debe trabajar con grupos numerosos de alumnos, a fin de generar condiciones suficientes para el desarrollo de competencias generales y específicas que demanda su perfil profesional.

Por cierto, no se debe olvidar que los medios, recursos y procedimientos de enseñanza han servido de apoyo para aumentar la efectividad del trabajo del profesor. Situación que no permite sustituir la función formativa, educativa y humana del profesor; sino que es un factor que ayuda a racionalizar la carga de trabajo de los estudiantes y el tiempo necesario para su formación científica. Además, estos recursos didácticos permiten mantener y/o elevar la motivación hacia la enseñanza y el aprendizaje, tanto de los alumnos, como del profesor.

En suma, hay que reconocer que la influencia que ejercen recursos, los medios y procedimientos didácticos en la formación y desarrollo de competencias personales, instrumentales y transversales de los alumnos pueden ser factores diferenciadores en un proceso de formación profesional superior. Estas herramientas didácticas permiten activar funciones cognitivas, socio-emocionales y procedimentales en la adquisición del conocimiento; también, reducen el tiempo dedicado al aprendizaje y la enseñanza, dado que objetivan los contenidos y 
propósitos del proceso enseñanza - aprendizaje, para facilitar la asimilación de lo esencial de la acción educadora.

\section{El mundo universitario en el contexto actual}

El nuevo escenario de la globalización plantea desafíos inéditos para el mundo universitario. Los acuerdos establecidos por los países europeos, a partir de la Declaración de Bolonia, donde se establecen objetivos comunes para toda la región europea y, posteriormente se adaptan a la realidad latinoamericana aplicado, terminan con la idea del tradicional aislamiento de las instituciones universitarias, para convertirlas en pilares fundamentales del proceso de desarrollo de estrategias políticas y productivas que ayudan al progreso científico, económico, cultural y social de las naciones y sus respectivas poblaciones.

La educación superior tiene la responsabilidad mayor de preparar a los estudiantes universitarios mediante el ofrecimiento de actividades curriculares, metodológicas y extra curriculares que contribuyan a su desarrollo integral como futuro profesional. Por ende, la academia debe realizar en forma permanente los cambios curriculares y didácticos necesarios para atender las necesidades de los individuos que reciben educación formal. Estos cambios deben ser capaces de responder esencialmente a situaciones vinculadas a:

- Los tipos y estrategias de aprendizaje que traen los nuevos alumnos de esta sociedad telemática, informatizada y multicultural, es decir, reconocer la diversidad de maneras que tiene los individuos para aprender.

- Un variado origen socio-cultural y procedencia escolar en la composición del alumnado universitario que dificulta la utilización de clases homogéneas, uniformes y tradicionales en su formación profesional.

- Las tendencias educativas modernas sobre el ideal de aprendizaje a lo largo de la vida (LLL) y la oferta de programas de educación permanente, referidos a post grado (diplomado, magister y doctorado) y al post-título (especialización y actualización profesional).

- Las expectativas de una sociedad competitiva sobre la universidad como instituciones de formación de capital humano avanzado, a fin de mantener los niveles de desarrollo tecno - socio - cultural del tiempo presente.

- La existencia de una tecnología compleja, diversa y sofisticada, que posibilita la virtualización del saber y el surgimiento de una economía del conocimiento, entre otras expresiones actuales que impactan en el quehacer formativo universitario. 
- Los cambios en los Perfiles Profesionales y los desafíos que conlleva el desarrollo de estas nuevas competencias y necesidades de formación que tiene el alumno, para potenciar la empleabilidad de los egresados universitarios en este cambiante y dinámico mercado laboral actual.

- El aumento en la competencia académica entre las instituciones de educación superior pública y privada, entre universidades locales y globales, para responder a la velocidad y cambio del conocimiento que propicia el actual modelo de desarrollo económico imperante en el país y mundo entero.

En suma, este nuevo escenario de la educación superior coloca a la Universidad como eje central de los actuales procesos asociados a la globalización y mundialización de la cultura, ciencia y tecnología occidental.

Por cierto, las nuevas técnicas e innovaciones didácticas y curriculares son reflejo de este fenómeno mundial, donde las tendencias educativas, como: la educación a distancia, la revisión de los procesos educativos, la certificación y acreditación de universidades, programas y carreras, la permanente investigación sobre procesos didácticos en distintos niveles de enseñanza, la formación de comunidades de aprendizaje (ya sea de naturaleza como virtual y/o la formación de redes de académicos) que promueven el aprendizaje colaborativo, entre otras expresiones educativas contemporáneas, que ayudan a dimensionar el desafío que tienen las instituciones educativas para adaptarse a este siglo XXI.

De igual forma, se debe reconocer el predominio del Paradigma del Aprendizaje en la labor educativa, al reconocer en el alumno, un descubridor activo y constructor de su propio conocimiento; lugar, donde el profesor asume un rol de orientador, de guía en la aventura del aprender. De esta forma, el agente principal del proceso enseñanza - aprendizaje es el aprendiz, cuya labor consiste en apropiarse y/o construir marcos conceptuales que son creados o propiciados por el profesor, a fin de responder a desafíos problemáticos y complejos que supone su propia formación profesional y personal para un escenario futuro, altamente competitivo y complejo para el mundo del trabajo.

Frente a esta situación, el docente universitario se enfrenta al desafío de pensar la enseñanza, desde la perspectiva del aprendizaje. Situación aún más compleja para el profesor universitario, cuando no tiene formación pedagógica y se desenvuelve en universidades regionales del país.

En definitiva, el actual mundo de la universidad requiere de un profesor que posea nuevas competencias y habilidades pedagógicas para poder responder a los desafíos de una sociedad global, que demanda profesionales autónomos, con 
flexibilidad de pensamiento y capacidad para adaptarse a situaciones propias de un entorno cambiante, como lo es la actual sociedad mundializada del siglo XXI.

\section{El "espacio abierto" (open space technology, ost) como metodología dialógica}

En la actualidad, el Espacio Abierto es reconocido como uno de los enfoques más innovadores para aprovechar de forma más eficiente las reuniones de trabajo, aportándoles un mayor grado de creatividad y productividad. A través de esta metodología es posible organizar reuniones con pequeños y grandes grupos (10 a 1000 personas) y mejorar las comunicaciones entre sus asistentes de una manera simple y efectiva, al centrar en los sujetos participantes el éxito de la reunión y darles la responsabilidad de crear una experiencia valiosa. (OWEN, 1992)

\subsection{Conceptualización del constructo de Espacio Abierto como técnica investigativa y recurso didáctico}

El Espacio Abierto (OST: Open Space Technology) es un procedimiento técnico implementado por Harrison Owen, con la finalidad de organizar la confrontación reflexiva sobre problemas complejos y abiertos, que permita a sus participantes asumir eventuales decisiones y proponer soluciones innovadoras y creativas a problemas cotidianos de sus integrantes. Tiene una estructura muy informal y abierta, con el objeto de gobernar los conflictos entre puntos de vista muy diversos, para encontrar soluciones oportunas a un problema serio y complejo.

La Técnica del Espacio Abierto es una forma de permitir a todo tipo de personas de cualquier organización poner en marcha reuniones, encuentros y eventos creativos. Es una práctica que se viene utilizando desde hace 20 años en todo el mundo. Los participantes crean y gestionan sus propias agendas y sesiones de trabajo sobre un tema concreto, incluso de importancia estratégica. Por ejemplo: ¿cómo poner en marcha un plan de formación para el voluntariado Regional?

El Espacio Abierto tiene reglas simples, pero efectivas, con las que crea un ambiente similar al que se da durante los recesos para tomar "el refrigerio" en un evento. La incertidumbre creada por el "aparente" caos proveen la libertad para crear, y el estado de apertura que se genera permite abordar mejor los temas y dar una mejor dirección. El Espacio Abierto no es sólo un evento, es una nueva forma de trabajar, de pensar, de reunirse, que puede continuar más 
allá de las reuniones. El trabajo más productivo se da cuando se crea un clima que favorece la iniciativa y el aprendizaje.

Puede ser llevado a cabo con grupos de 10 a 1.000 personas, trabajando en sesiones de un día, asambleas de tres o una reunión semanal de los miembros de un equipo. Aunque como espacio abierto, queda la posibilidad de modificar estos criterios.

En esta perspectiva, muchas de estas características se parecen a una sala de clases, con un numeroso número de alumnos, organizado en grupos y sub grupos, con desorden y ruidos que interfieren el trabajo docente. En fin, generando mayores problemas de control al profesor y una dificultad creciente para el propio aprendizaje de los estudiantes; situación muy estresante para el docente comprometido con una educación de calidad. Estas características externas que posee el Espacio Abierto también se corresponden con el ideal de lograr un aprendizaje significativo en los alumnos universitarios.

A un nivel más profundo, el Espacio Abierto permite a las personas experimentar una organización de calidad distinta, en la cual los grupos de trabajo son autogestionados, el liderazgo compartido, la diversidad de sus participantes se convierte en un recurso y no en un problema; es una experiencia compartida que brinda la oportunidad para un cambio organizativo fundamental. Proporciona una estructura que genera energía, compromiso y liderazgo sin necesidad de complicados procedimientos logísticos ni de planeación por anticipado. Los organizadores del evento crean un tema suficientemente fuerte que atrae poderosamente el interés de la gente, estimula una amplia discusión y permite el desarrollo de tópicos que los participantes consideran de importancia.

En otras palabras, el Espacio Abierto permite encontrar nuevas ideas para potenciar y/o cambiar instituciones, para enriquecer la formación profesional recibida, para investigar un contexto socio-cultural dado, para desarrollar competencias genéricas o transversales, etc. De igual forma, se puede pensar que su posible aplicación como técnica didáctica permitiría dinamizar el trabajo docente con grandes grupos de alumnos, sobre todo a nivel de los primeros años de estudios universitarios.

Esta doble condición que tiene el Espacio abierto como técnica investigativa y como técnica didáctica, permite al profesor hacer investigación al interior del aula y ser una herramienta que ayuda a mejorar sus clases, al dar mayor protagonismo al todos los integrantes de su curso.

En suma, la OST es una técnica participativa, cuya aplicación en carreras universitarias, organizaciones o en grupos sociales, permite el logro de resultados superiores, es decir, más ambiciosos, con alto grado de compromiso individual, 
grupal, o de toda la organización involucrada, y sobre todo, en forma rápida y económica para el docente - investigador.

\subsection{Forma de empleo: una propuesta}

El Espacio Abierto funciona mejor cuando el trabajo a realizar es complejo, las personas y las ideas diversas, la necesidad de resolución de conflictos es grande y disponemos de poco tiempo. Consigue de una forma sencilla y eficaz que la gente y las organizaciones se pongan en acción cuándo y dónde sea necesario.

Aparentemente en el Espacio Abierto hay falta de estructura e improvisación, pero no es así, realmente está muy estructurado: se ajusta a la gente y al trabajo con tanta perfección, que pasa desapercibido.

En la vida académica, para muchos profesores universitarios la asistencia a seminarios, congresos, cursos, encuentros etc., representa la oportunidad de establecer o fortalecer contactos personales o institucionales. Por cierto, a través de esta situación se pone de manifiesto la esencia de OST, es decir, crear un ambiente y el momento para que las personas digan y hagan aquello que les interesa, lo que les conviene, les apasiona y que les genera un compromiso responsable e incondicional con sus propias expectativas.

De igual forma, la sala de clases puede ser un buen espacio para aplicar el espacio abierto, dado que muchas características que supone la aplicación del OST se encuentran presentes durante las aulas universitarias. Sin embargo, el gran problema para su aplicación didáctica lo constituye la elección de la problemática a examinar por los alumnos y sus criterios de evaluación.

\subsection{Preparación y ejecución}

\subsubsection{Rol del coordinador - Profesor:}

El profesor coordinador ayuda a los alumnos a clarificar el motivo del encuentro, da a conocer los objetivos y procedimientos del trabajo por hacer, la forma de participar y clarifica todas las dudas de los alumnos participantes.

Al mismo tiempo, el docente se asegura que la planificación del Espacio Abierto sea en la mejor fecha y hora dada. Durante su ejecución, el profesor debe asesorar a los participantes y/o tener un equipo de ayudantes que apoye su realización, ya sea solucionando dudas, asesorado a cada grupo según sus dificultades o apoyando la redacción de los informes parciales. Todo lo cual 
debe permitir la elaboración del informe final de los resultados obtenidos con la aplicación del OST, en dicho curso.

Cabe señalar que la aplicación de la técnica del Open Space requiere que el profesor coordinador desarrolle en forma previa a la reunión grupal, algunas actividades de planificación, tales como:

Tener fijado con anticipación, el lugar, día y hora de la reunión. Asimismo, haber enviado las recordaciones (en formato de invitaciones) con la debida antelación y haber efectuado la suficiente confirmación o recordatorio de dicho evento a sus participantes. Se pretende que todo el curso este presente en esta actividad grupal.

a) Examinar la composición de la muestra invitada, a fin de garantizar los resultados esperados.

b) Tener preparado el equipo necesario para la reunión: sillas y mesas, pizarrones, computador y data show, lápices y hojas para escribir, grabadora, máquina fotográfica digital, etc.

c) Tener un guión del desarrollo de la reunión que permita alcanzar sus propósitos establecidos, ya sea la discusión colectiva, el listado de preguntas abordadas, el tipo de participación de los asistentes, el registro de su desarrollo y las conclusiones alcanzadas, entre otros aspectos.

A continuación, se presenta una Lista de Verificación del Enfoque de Espacio Abierto para el profesor coordinador.

- $\quad$ Pregunta fundamental: ¿La tecnología de Espacio Abierto es la más adecuada para nuestros propósitos pedagógicos?

- Tema: ¿Es claro, preciso y con suficiente espacio para la imaginación?

- Invitación: ¿Nuestros alumnos invitados tienen la suficiente información que les permita llegar a la hora precisa, listos para trabajar?

- Tiempo: ¿Hemos asignado suficiente tiempo para lo que queremos hacer?

- Espacio Principal: ¿Nuestro salón de reuniones es suficientemente espacioso para hacer un círculo cómodo?

- La Pared: ¿Tenemos una pared descubierta en nuestro salón de reuniones que todos puedan ver?

- Otros Espacios: ¿Tenemos suficientes espacios o salones pequeños (10 salones para 200 participantes, por ejemplo) para que todos puedan ubicarse cómodamente? 
- Comida y Bebida: ¿Hay posibilidades de ofrecer algún tipo de comidas y refrigerios?

- Consulta previa con los participantes y la autoridad: ¿Hemos dedicado suficiente tiempo a los participantes y a la autoridad (jefe de carrera, director, etc.) para explicarle la técnica del Espacio Abierto y nuestras necesidades para dicho evento?

\subsubsection{El proceso del Espacio Abierto}

\section{- Presentación}

Los alumnos participantes se reúnen para la sesión plenaria de apertura, es decir, el momento en el que se dan las instrucciones de trabajo. Los participantes pueden sentarse en círculo como corresponde a una metodología de trabajo abierta y participativa. Una persona que asume el rol de coordinador o asesor señala el tema a tratar, describe los principios de la técnica y su gestión en cada grupo o sub grupo.

\section{- Cuatro principios básicos:}

- Cualquier persona que llegue es bienvenida. Se ha invitado a todas las personas claves para lograr el objetivo propuesto. Cuando la reunión comienza las personas que han llegado, por definición, son las personas apropiadas por cualquier razón que hayan elegido venir.

- Lo que suceda es lo único que pudo suceder. Los participantes están limitados sólo por sus expectativas, de manera que es mejor no preocuparse por lo que pudo haber sucedido.

- Cualquier hora en que se comience es la hora adecuada. La observación del reloj puede inhibir la creatividad y el aprendizaje. Todos deben hacerse responsables de su tiempo y de sus aportes.

- Cuando se termina se termina. El proceso creativo y los mismos grupos tienen sus propios ritmos, a veces se usa más tiempo o menos tiempo del que se esperaba.

En suma, el punto clave durante la reunión de esta metodología radica en la autoorganización, es decir, que cada persona elige formular y trabajar el tema más le interesa y por ende, puede encontrar grupos afines y con una alta motivación por para llevar algo a cabo. 


\section{-Creación de la agenda}

Cualquiera que se sienta inspirado puede ofrecer una o más temáticas por debatir, por ejemplo (un taller sobre habilidades sociales, un grupo de debate sobre cómo mejorar la comunicación de las organizaciones, una tarea como la elaboración de una guía de buenas prácticas), etc. Para ello, creará un simple póster o escribirá en un papel graf, el título de la sesión y su nombre, ofrecerá una pequeña explicación al grupo y finalmente, elegirá un espacio físico y delimitará el tiempo de realización.

Ahora bien, este reconocimiento a la posibilidad de auto-organización debe ser entendida dentro de la agenda o tema central que propicia el profesor para la aplicación del OST en dicho curso. De lo contrario, no tener claro el tema central de trabajo por realizar, dificultad la efectividad de su aplicación.

Por tanto, el profesor y/o coordinador de cada grupo deberá proponer una agenda temática que invite a la reflexión y discusión entre los participantes, dentro de los límites temáticos propuestos para dicha sesión de aprendizaje.

\section{- Inscripción}

Los afiches o temas de las diferentes propuestas se colocan en la pared o en el suelo y los participantes se inscriben en la sesión o sesiones que deseen. Por cierto, el uso de diferentes colores hace el ejercicio mucho más visual. En esta fase, se produce la mayor parte de la negociación, por ejemplo los participantes con propuestas de temas similares pueden decidir formar grupos o unir fuerzas. También, puede pedirse que las sesiones se ajusten en el tiempo, para hacer posible una participación en varios temas a lo largo del tiempo destinado.

En este caso y durante la sesión, el profesor y/o coordinador de los grupos puede estimular la participación de todos sus asistentes, con la nominación de los diferentes grupos de estudios que, previamente, ha planificado con sus ayudantes de investigación.

\section{- Sesiones}

Interesa destacar que el trabajo de análisis y reflexión grupal debe culminar en el tiempo dado para la reunión. El profesor coordinador deberá cautelar este aspecto para el éxito del Espacio Abierto (OST).

El OST como técnica investigativa, son los organizadores del evento que deciden quién participa, generalmente agentes sociales, educativos, culturales, etc. y de todos los niveles posibles de una organización. En este caso, fueron académicos de diferentes unidades y facultades, disciplinas y trayectorias profesionales. 
En cambio, el OST como técnica didáctica, el profesor coordinador invita a todo su curso a participar, promoviendo una asistencia completa para dicha actividad, buscando que las inasistencias sean las mínimas. Solo es necesario advertir sobre la temática central de la reunión grupal y las condiciones de la asistencia: puntualidad, interés, entusiasmo, etc.

En cuanto al lugar físico para su realización, tanto espacio como sea posible será bienvenido. Se recomienda, un salón plenario o aula grande con sillas móviles para ponerlas en círculo. Otros espacios más pequeños para las sesiones serán también son necesarios. Un computador por cada 20 participantes y una impresora. Papel, bolígrafos, papel continuo o papeles de colores, pegamento, destacadores, etc. Pueden ayudar a exito del evento.

\section{- Informe de la sesión y reflexión final}

Para la conclusión y cierre de la sesión, cada coordinador de los sub-grupos debe resumir los puntos principales y las acciones acordadas, las cuales pasan a ser parte de las conclusiones del evento. El resumen y la compilación de los informes o relatos deben hacerse en el mismo evento, de modo que cada uno de los participantes pueda llevar las conclusiones consigo. Finalmente, los participantes tienen la oportunidad de comentar brevemente sobre lo que han aprendido, como una forma de cerrar el Espacio Abierto. El plenario termina con una sesión final o espacio cerrado en la cual los participantes reflexionan sobre lo ocurrido.

Al final del evento, los participantes reciben un reporte escrito con una lista de aquellas personas que participaron en cada grupo. Esta información apoya el desarrollo de diversas redes y/o grupos de interés. Se definen las prioridades, se desarrolla un plan de acción y los involucrados son identificados. Los participantes se llevan nuevas reflexiones y la sensación de haber avanzado en su propio auto-conocimiento.

Para alcanzar este resultado, el profesor coordinador debe tener planificado los tiempos de desarrollo, discusión y elaboración del informe final del OST. Se sugiere, la presencia de alumnos ayudante cuando el grupo sea demasiado grande o cuando se aplica por primera vez, a fin de disminuir los errores o dificultades que puedan surgir en su desarrollo.

\section{- Esquema del informe final de la sesión Espacio Abierto}

Desde el punto de una investigación, el coordinador para elaborar el informe de lo acontecido, deberá responder a los siguientes aspectos: 
- Identificar el tema central abordado y los diferentes sub-temas o problemas trabajados en cada sub-grupo.

- Nombrar a los líderes de cada grupo de trabajo y aquellos que asumieron un rol protagónico en la discusión global.

- Elaborar un listado de todos los participantes, ya sea por grupo de trabajo y tema analizado.

- Describir las razones sobre el interés por el tema, sus conclusiones y desafíos planteados.

- Relatar la sesión de trabajo: interacciones, soluciones, perspectivas de análisis y todo aspecto que ayude a comprender el trabajo desarrollado.

Desde el punto de vista de la didáctica, el profesor deberá responder a las siguientes interrogantes:

- ¿Las conclusiones obtenidas y/o resultados son reflejos de un proceso de aprendizaje de calidad?

- ¿Todos los alumnos participaron en las distantes etapas del OST?

- ¿Se dio oportunidad para desarrollar competencias genéricas (trabajo en equipo, iniciativa, responsabilidad, resolución de problemas, etc.) entre los participantes?

- ¿El aprendizaje logrado tuvo una naturaleza colaborativa y cooperativa para todos?

Por cierto, en ambas situaciones será el profesor - coordinador - investigador, quien determine el contenido y naturaleza de las interrogantes que permitan evaluar la eficacia de esta técnica investigativa - didáctica aplicada a su respectivo grupo.

\section{- Fortalezas y limitaciones del Espacio Abierto}

Como toda metodología de trabajo de naturaleza participativa y dialógica, el "Espacio Abierto" presenta fortalezas y potenciales limitaciones, tanto técnica investigativa como procedimiento didáctico. En cuanto a sus principales fortalezas, cabe advertir que:

a) Proporciona un ambiente seguro e inclusivo, para que la diversidad de participantes puedan intercambiar información, puntos de vista y estrategias sobre el desarrollo o situación problemática dada.

b) Asigna responsabilidad a los participantes, estimula la confianza, el liderazgo compartido y el compromiso a todo lo largo del proceso. 
c) Logra la participación a un bajo costo y de un modo no complicado, contando con el interés de los propios participantes, no necesariamente entrenados ni en posiciones de liderazgo en las instituciones involucradas.

Por otra parte, algunas posibles limitaciones son:

a) La estructura flexible del Espacio Abierto puede afectar la representatividad del grupo, cuando no asisten actores claves para el grupo o profesor.

b) No hay garantía de que los temas que el profesor coordinador cree que son los importantes se incluyan en el programa, pues son los participantes los encargados de la selección de tópicos o materias a discutir.

c) Algunas sesiones pueden terminar con muy pocos o demasiados participantes, porque no se establecen límites con respecto a la demanda.

En síntesis, el Espacio Abierto es un enfoque apto para explorar temas, planear el futuro, estructurar rápidamente, construir y fortalecer equipos, mejorar las comunicaciones y energizar organizaciones. No debe usarse si se piensa que ya se saben las respuestas de antemano. El líder del grupo debe ser consciente que está creando un espacio para la iniciativa, y por lo tanto debe estar preparado para lo inesperado y estar abierto al cambio. Su intención debe ser transparente, el tema debe ser presentado de manera clara y respondiendo a un real interés para todos los participantes.

De igual forma, esta técnica puede ofrecer mayores posibilidades de mejorar la docencia tradicional en cursos numerosos de alumnos, al permitir y fortalecer el desarrollo personal y social de todos los participantes. Muchas de las características que tiene el Espacio Abierto se reproducen en la sala de clases, por tanto, no es necesario hacer una gran adaptación para ser utilizada como técnica didáctica en la formación profesional superior.

\section{Aplicación piloto del espacio abierto \\ en dos universidades regionales chilenas}

Al describir esta metodología técnica de indagación social se busca entender su aplicación en el presente proyecto de investigación denominado: "La construcción del sujeto docente universitario en universidades regionales: factores claves en su desempeño académico y profesional", financiado por el gobierno de Chile, en la línea Conicyt/Fondecyt, cuyos objetivos están relacionados con la construcción del sujeto docente universitario en universidades locales.

De igual forma, este articulo presenta una descripción de esta técnica investigativa que ayude a comprender su potencial aplicación como una innova- 
ción didáctica que, eventualmente, se puede transformar en un procedimiento didáctico habitual para la enseñanza profesional superior.

A continuación, se presenta una breve caracterización de los resultados obtenidos en dos universidades regionales, pertenecientes al Consejo de Rectores, en las cuales se llevó a cabo la citada metodología: Una, ubicada en el centro de Chile, cuya universidad es representativa de la región y la otra, situada en la zona sur del país, cuya fundación no sobrepasa los 20 años. Ambas universidades tiene todas las actividades y funciones que caracterizan a una universidad nacional.

La organización del Open Space se inició con una serie de reuniones semanales, que fueron cumpliendo diferentes objetivos en el cronograma de trabajo propuesto durante el desarrollo del citado proyecto de investigación. A la vez, teniendo en consideración las características y procedimientos que supone la aplicación del Espacio Abierto como técnica investigativa y didáctica.

\section{a) Preparación y ejecución:}

\section{- Convocatoria}

Una invitación vía digital fue extensiva a una muestra de docentes de ambas universidades regionales.

\section{- Preguntas base}

En lo que concierne a las preguntas de base para la realización del Espacio Abierto, éstas se articularon en torno a seis dimensiones que fueron las siguientes:
a) Curriculum
b) Gestión universitaria
c) Evaluación docente
d) Evaluación de estudiantes
e) Innovación
f) Preparación en docencia universitaria
g) Responsabilidad social

Al tener presente dichas dimensiones, fue más fácil la elaboración y redacción definitiva de las preguntas "gatilladoras" del Espacio Abierto, las cuales se aplicaron en esta posterior jornada.

\section{- Preparación del profesor coordinador institucional}

El equipo de proyecto tuvo reuniones con los coordinadores institucionales de ambas universidades para preparar su desempeño y labor coordinada con 
los otros participantes del proyecto. El propósito de dicha preparación fue obtener una labor semejante en ambas universidades, para validar los resultados obtenidos por un tercer integrante: un "secretario" (véase Anexo B: Plantilla).

En caso de ser aplicada como técnica didáctica, dicha labor la cumple el profesor de aula y sus eventuales ayudantes.

\section{b) Realización del Open Space}

El día 9 de Septiembre de 2008 asistieron 25 docentes, de diversas disciplinas y facultades de la Universidad central, quienes, previa entrega de carpetas y breve explicación acerca de la actividad, procedieron a escoger voluntariamente el tema de interés. Se formaron cinco grupos, con un número de integrantes que fue de 2 a 6 personas. Un grupo tomó dos temas: Currículo y Evaluación Docente. El único tema que no fue tratado fue el de Responsabilidad Social. Para la discusión de las preguntas, se dio un tiempo fijo de 1 hora. Posteriormente, un integrante de cada grupo, presentó las conclusiones en aproximadamente 5 minutos, a la vez que otro integrante fijaba en un cartel, sus conclusiones de esta actividad.

De igual modo, este procedimiento fue realizado en la Universidad de la zona del sur del país, con un número similar de participantes y temáticas dadas.

\section{c) Cierre}

Al finalizar la actividad, se diól la oportunidad a que los docentes participantes opinaran acerca de la experiencia vivida. Fue unánime el encontrar una buena iniciativa, reconociendo principalmente la oportunidad de intercambiar experiencias con colegas de diferentes áreas, así como la oportunidad de tener una instancia de reflexión acerca de la docencia en la Universidad. También se mostraron muy dispuestos a repetir la actividad en un momento de menor actividad académica.

Se tomó el acuerdo de enviar vía correo electrónico a sus participantes, las conclusiones del Open Space, algunas de las cuales se detallan a continuación.

\section{d) Resultados e interpretación de su aplicación como técnica investigativa}

En este primer análisis de resultados, se ha focalizado la interpretación en algunas preguntas que son representativas de las dimensiones consideradas en la aplicación de Open Space, para una muestra intencionada de docentes universitarios de ambas universidades participantes de la presente investigación.

Frente a la pregunta: ¿Cómo mejorar la enseñanza universitaria?, se obtuvieron algunas respuestas sobre: 
- Mejorar la capacidad docente de los profesores, los profesores son especialistas en su tema, pero con dificultades. a veces importantes, en la forma de entregar y transmitir el conocimiento. Esto puede lograrse a través de cursos o talleres.

- Dar a la docencia a la docencia la importancia que tiene, eso significa mejorar la forma de evaluar la docencia de pregrado.

- Creo que además debería existir perfeccionamiento permanente de los académicos en este tema. Con respecto a los recursos, considero que son necesarios, pero no constituyen un impedimento infranqueable para realizar mejoras a la enseñanza, aunque sea a una escala pequeña.

- También pienso que es necesario que la universidad valore la dedicación a la docencia, pues actualmente no lo hace, o al menos tiene una valoración menor a la investigación en ciencia básica o aplicada. Eso a veces determina que gente capacitada e interesada en docencia tome un rumbo diferente.

- Para mejorar la docencia pienso que habría que crear más instancias para el perfeccionamiento, más cursos y talleres organizados por la Universidad. Tal vez crear fondos para financiar o co-financiar magísteres y diplomados en educación en instituciones externas a quienes estén comprometidos con la docencia. Pienso que el perfeccionamiento es fundamental, pues la mayoría llegamos por la competencia en la disciplina especifica y hacemos clases sin sabe nada de educación.

- Considero que los espacios existen, pero son pocos y, lamentablemente no todos los académicos se interesan en participar. Creo que se deberían realizar jornadas de reflexión en torno a la docencia universitaria que fueran OBLIGATORIAS para todo académico que participe en actividades de docencia, o al menos, para aquellos que tienen alguna responsabilidad a este nivel.

Por cierto, resulta importante destacar que el tema de la enseñanza universitaria es el eje central del desarrollo estratégico de una universidad, donde sus profesores son el factor clave para la transformación de una institución de educación superior. Por lo cual, la Universidad debe crear espacios y actividades de acompañamiento pedagógico que permita potenciar las innovaciones curriculares y didáctica que las actuales tendencias mundiales reclaman de la educación superior. 
De igual forma, frente a la pregunta: ¿Cómo potenciar la formación en docencia universitaria? Se obtuvieron algunas respuestas significativas que se expresan en seguida.

Dar reconocimiento suficiente y significativo a las actividades de docenci.

Aprovechar oportunidades de procesos de re-acreditación y cambio curricular; además de la competencia externa (otras universidades).

Fortalecer la Dirección de Docencia.

Darle una relevancia similar a la excelencia en la investigación.

Formar y/o fortalecer grupos de docencia en cada facultad y también en los departamentos.

[...] a través de actividades formales: Permanentes, estructuradas, secuenciales, extensivas a todos los profesores (todas las jerarquías), con énfasis en la formación pedagógica. La iniciativa debe ser liderada por la propia universidad. Considerando también las particularidades de cada Facultad.

Con respecto a la evaluación, en general no estoy de acuerdo con ella. La mayoría aplica sólo pruebas con alternativas y verdadero y falso. Aunque se apliquen pruebas con combinaciones de instrumentos, siguen siendo clásicas. Considero que debido a la falta de motivación y los cambios ya mencionados en los estudiantes es necesario incorporar otras situaciones evaluativos.

Para utilizar otros instrumentos de evaluación que permitan evaluar conocimiento profundo y competencias es necesario: revisar los contenidos de las asignaturas a los requerimientos actuales; aumentar el número de profesores por asignatura y (saber) como evaluar por competencias.

En general, los profesores manifestaron gran interés por el tema de la docencia universitaria y la valoraron tanto como la investigación. En los últimos años, algunos profesores se han capacitado en temas pedagógicos, (participación en Magíster, Diplomados, cursos de capacitación, talleres de actualización), no obstante, aún manifiestan sentirse sin las herramientas suficientes para realizar una buena docencia. Pero, es el área de Evaluación de los Aprendizajes uno de los puntos críticos, ya que no se sienten suficientemente capacitados al momento de evaluar a sus estudiantes. 
Tal vez, un aspecto interesante de mencionar lo sea el concepto de universidad regional por sus implicancias que tiene para la docencia universitaria, es decir: ¿Cómo potenciar una Universidad regional? Interrogante que provocó una serie de respuestas, tales como:

Fortalecer la Universidad estableciendo una línea, un sello que permita reconocer esta universidad, para eso se deberían potenciar las áreas más fuertes, que nos distinguen dentro de la región, en este caso la investigación en ciencia básica y aplicada.

Desarrollar un hospital clínico asociado a un centro de biomedicina de primer nivel que permita que las personas no concurran a Sgto. Este centro debería estar asociado a un centro de Investigación en Biomedicina.

Se debe lograr que por parte del gobierno exista un fondo para el desarrollo de las universidades tradicionales.

Creo que la universidad está respondiendo a lo que el mercado quiere, es así que es el mercado que ha determinado la creación de nuevas carreras, el aumento de cupo en algunas carreras. Habría que revisar en forma crítica los requerimientos de estos profesionales para adecuar las mallas curriculares.

Para potenciar la formación en docencia, reitero la idea de generar más instancias para el perfeccionamiento y la creación de un fondo para perfeccionamiento de académicos en docencia universitaria en instituciones externas, ya que seguramente algunos estaríamos dispuestos a realizar una formación sería y formal fuera de horario, si no fuera por motivos económicos.

Concuerdo con una capacitación obligatoria y más importante aún, continua, pues los alumnos, los medios y las tecnologías van cambiando y idealmente uno se debería ir a adaptando a ellos. Eso debiera ser controlado y valorado en la evaluación del académico (por la universidad).

De igual forma, debería incorporarse tecnología en las clases, "porque facilita el proceso de enseñanza-aprendizaje, por ejemplo a través de clases interactivas a través de plataformas, con animaciones, videos, evaluaciones a través de e-learning ( $P / E$ moodle), actividades interactivas tales como elaboración de caricaturas, juegos bursátiles 
y museos. Deberían involucrarse todos los profesores y no sólo los de jornada completa.

Al evaluar dicha pregunta se percibe que la idea de una universidad regional se encuentra asociada al impacto socio-económico y cultural que provoca su actividad, donde la formación de profesionales ocupa un lugar destacado, al igual que la investigación de frontera. Este desafío se logra cuando existe una masa crítica de docente predispuesto al cambio pedagógico y didáctico que supone la actual sociedad del conocimiento.

Un análisis más específico sobre la docencia universitaria conlleva a preguntarse: ¿Es necesario planificar la docencia (asignaturas)?. Algunas respuestas obtenidas fueron:

Es necesario y aplicar la adecuada gestión para el cumplimiento.

Me parece obvio que debe haber una adecuada planificación, sin embargo, muchas veces parece que ésta no se ha realizado o ha sido deficiente. Considero que, especialmente en carreras que cursan asignaturas en más de una facultad, debería existir una mayor y mejor coordinación para diseñar las mallas curriculares. Esto puede deberse a una mala comunicación inter-facultad.

Creo que debería haber una revisión de algunas mallas curriculares que he visto últimamente, pues no se corresponden con las competencias que debemos entregar a nuestros estudiantes. Una posibilidad para mantener las mallas actualizadas sería formar una comisión (lo más representativa posible) que se reuniera una vez al año para evaluar el funcionamiento de dicha malla.

De esta forma, se percibe que la gestión pedagógica es una necesidad real del docente universitario para actualizar su labor y dar cumplimiento a las exigencias que deriva del nuevo modelo de competencias que esta siendo aplicado en la enseñanza universitaria. Además de una adecuada planificación docente, se requieren nuevas herramientas y espacios para adecuar la labor del docente universitario al enfoque basado en competencias que está siendo vivenciado en el actual sistema nacional de formación profesional superior.

En suma, estos resultados obtenidos permiten destacar su potencial para describir problemas y sus posibles soluciones en el ámbito académico. De este modo, el Espacio Abierto se advierte como un valioso recurso para organizar actividades de formación, talleres, grupos de trabajo, encuentros de organiza- 
ciones o cualquier tipo de reunión, donde el tiempo sea un recurso escaso y la creatividad, un ingrediente necesario. Situación que no fue ajena a la presente experiencia desarrollada en ambas universidades.

\section{e) El Espacio Abierto como técnica didáctica}

Lo expresado permite inferir que la técnica del Espacio Abierto se basa en la autoorganización de las personas participantes y en la conformación de grupos que permitan mejorar la comprensión de situaciones comunes para generar soluciones nuevas a dichas problemáticas. Así, por ejemplo, todas las personas participantes en el encuentro plantearon una "pregunta” sobre alguna cuestión de su interés.

De dicho análisis, se extrajeron algunas “áreas de interés”, que fueron tratadas por las personas que así lo deseaban en espacios grupales que se habilitaron para ello.

Esta es la lógica básica de la metodología de trabajo que propicia el Espacio Abierto, una forma poderosa y sencilla de hacer que las personas y organizaciones se muevan cuando y donde sea necesario. No está demás de señalar que esta situación se puede aplicar a aula de clases, sobre todo en la formación profesional, cuando los estudiantes universitarios necesitan adquirir un mayor protagonismo en su propio aprendizaje y buscar desarrollar estrategias tendientes a lograr su propio desarrollo personal y profesional para asegurar su mejor inserción laboral.

De este modo, el Espacio Abierto funciona mejor cuando el trabajo que se debe realizar es complejo, las personas y las ideas que están involucradas son diversas, la pasión por resolverlo (y potencial para conflictos) es enorme, y el tiempo para realizarlo es siempre escaso. Desafío muy propio del trabajo académico, cuando se debe impartir una formación profesional a una diversidad de estudiantes, tanta en su estilo de aprendizaje, motivación y valores, cuanta a su procedencia socio - cultural.

\section{Conclusiones y algunas interrogantes}

El espacio abierto es una técnica investigativa que también puede ser aplicada como innovación didáctica en la formación profesional superior. Ahora bien, si se revisa el desarrollo de una sesión de Open Space se descubre que los participantes se encuentran sentados en un círculo, donde además tienen la oportunidad de pegar en la pared o pizarra, los temas que sienten importantes, relacionados a un problema o tema principal que les es significativo y común. 
Por cierto, en el Espacio Abierto esto ocurre espontáneamente, pero también puede darse en una clase cualquiera.

Con un propósito claro, todos los participantes durante la primera parte de la reunión crean una agenda de temas en común y organizan grupos autodirigidos, después de la presentación general del coordinador del evento. Al tratar temas que son relevantes para quienes conforman el grupo de discusión, empieza a emerger un sentimiento de comunidad.

Cada uno de los temas de interés es tratado con igual importancia e interés por todos. ¿Qué mejor manera de llegar más lejos que empezar por lo que más le apasiona a uno? Todos los que comparten la pasión, el interés, o inquietud por algo deben juntarse para trabajar en ello. El proceso permite encontrar soluciones juntos y el ambiente seguro que se ha creado facilita una comunicación constructiva entre los participantes. La colaboración se hace intensa y atractiva y el potencial para asumir compromisos se incrementa porque los participantes se han apropiado del tema y de las oportunidades que emergen para su propio desarrollo profesional. Este hecho emerge con toda claridad en las muestras de ambas universidades consultadas.

Una lectura rápida de los resultados obtenidos en ambas universidades permite señalar lo siguiente:

- Los participantes comentan estar muy de acuerdo con este tipo de instancia.

- Los Docentes destacan la heterogeneidad del grupo, así se favorece el intercambio de opiniones de las distintas disciplinas, facultades y/o campus.

- Reconocen y critican la poca participación e interés de otros docentes en espacios o temas como estos.

- Destacan que la poca participación de otros docentes es un problema real para la universidad.

- Por último, los docentes presentan disposición para una nueva invitación de este tipo.

Finalmente, no hay que olvidar que el tema del presente proyecto es la acción pedagógica del profesor universitario y la problemática que conlleva su quehacer académico y profesional, en dos instituciones de enseñanza superior pertenecientes al consejo de rectores y ubicadas en regiones distintas del país. Situación descrita, de manera parcial, con la aplicación del Open Space y cuyos resultados permiten inferir su probable aplicación como técnica didáctica en la enseñanza superior. 
La reflexión individual y colectiva sobre la formación del docente universitario ayuda a mejorar la práctica pedagógica del profesor, es decir, crear instancias de diálogo, auto-examen e intercambio, contribuyen a socializar las innovaciones y experiencias en el campo de la didáctica universitaria. De este modo, incorporar nuevas metodologías didácticas, como es el caso del espacio abierto, puede potenciar el trabajo pedagógico del profesor universitario cuando se ve enfrentado en su trabajo cotidiano a grandes grupos de alumnos.

\section{Referencias}

HEINZ , Mandl; WINKLER, Katrin; SCHNURER, Katharina.

Instrumentos para la gestión del conocimiento. Estrategias organizacionales. Berlin: InWEnt - Capacity Building International;

Alemania. Cooperación Tecnológica, Desarrollo de Sistemas y Gestión de la Formación Profesional, n. 7, 2004.

HERMAN, Michael. En apoyo al mundo del espacio abierto. Disponible en: <http://www.openspaceworld.org>. Acceso en: 15 jan. 2009.

OWEN, Harrison. Coffee break produtivo. Usando o sucesso-sem-esforço da tecnologia do Espaço Aberto. Traducción del “User’s Guide”, de Alda Szlak-Freier, São Paulo: W11 Editores, 2003. Disponible en: <http://www. w11editores.com.br>. Acceso en: 15 jan. 2009.

OWEN, Harrison. Emerging order in open space. Disponible en: <http:// www.openspaceworld.org.>. Acceso en: 1 mar. 2009

OWEN, Harrison. Open space technology: a user's guide. Maryland: Abbott Publishing, 1992.

OWEN, Harrison.Tales from open space. Maryland: Abbott Publishing, 1995.

SILVA, Artur Ferreira da. Fundamentos da metodologia de reunião em espaço aberto. Cadernos INA, Oeiras, Potugal, n. 2, 2001. 


\section{ANEXOS:}

Anexo A:

\section{PREGUNTAS PARA EL OPEN SPACE}

\section{CURRICULUM}

1. ¿Cómo mejorar la enseñanza universitaria?

2. ¿Es necesario planificar la docencia (asignaturas)?

3. ¿Qué opinión le merece las actuales mallas curriculares?

\section{GESTION UNIVERSITARIA}

1. ¿Los Consejos de Carrera cumplen su función?

2. ¿Está de acuerdo con la formación que reciben sus estudiantes para su futuro desempeño laboral?

3. Existen espacios de análisis en torno a la docencia universitaria?

\section{EVALUACION DOCENTE}

1. ¿Debe un profesor Instructor hacer más docencia que investigación?

2. ¿¿La evaluación docente estimula la carrera académica?

3. Se siente satisfecho(a) con su condición de profesor universitario(a)?

\section{EVALUACION DE ESTUDIANTES}

1. ¿Son adecuadas las pruebas de selección múltiple como instrumento evaluativo?

2. ¿Ha notado diferencias en el rendimiento de los alumnos durante los últimos cinco años?

3. ¿Usted está de acuerdo con la forma de evaluar a sus estudiantes?

\section{INNOVACION}

1. ¿Incorporaría herramientas tecnológicas para su docencia?

2. ¿ ¿Su carrera estimula el desarrollo de la creatividad y la innovación en sus estudiantes?

\section{PREPARACION EN DOCENCIA UNIVERSITARIA}

1. ¿¿Debería ser obligatoria la capacitación en docencia universitaria?

2. ¿Cómo potenciar la formación en docencia universitaria?

3. ¿Cuál debería ser el perfil del docente universitario actual?

\section{RESPONSABILIDAD SOCIAL}

1. ¿Cómo potenciar una Universidad regional?

2. ¿ ¿Se están preparando los profesionales que la región requiere para su desarrollo?. 
Anexo B:

\section{PLANTILLA SECRETARIO(A) \\ ESPACIO ABIERTO "CONVERSANDO LA DOCENCIA".}

Martes 9 de Septiembre de 2008.

Con el fin de contrarrestar validez de las opiniones, le agradeceremos completar las columnas según corresponda.

TEMA ANALIZADO:

NOMBRE COORDINADOR(A):

NOMBRE SECRETARIO(A):

\begin{tabular}{|c|c|c|c|}
\hline $\begin{array}{c}\text { NOMBRE DE LOS } \\
\text { INTEGRANTES DEL } \\
\text { GRUPO }\end{array}$ & $\begin{array}{c}\text { ¿Quiénes } \\
\text { participaron más? }\end{array}$ & $\begin{array}{c}\text { ¿Quiénes fueron } \\
\text { más críticos? }\end{array}$ & $\begin{array}{c}\text { ¿Quiénes motivaron } \\
\text { la discusión? }\end{array}$ \\
\hline & & & \\
\hline & & & \\
\hline & & & \\
\hline & & & \\
\hline & & & \\
\hline & & & \\
\hline & & & \\
\hline & & & \\
\hline & & & \\
\hline & & & \\
\hline & & & \\
\hline & & & \\
\hline & & & \\
\hline
\end{tabular}

¿Qué otros temas que surgieron de la discusión se trataron? 